\title{
Broadly sampled orthologous groups of eukaryotic proteins for the phylogenetic study of plastid-bearing lineages
}

\author{
Mick Van Vlierberghe ${ }^{1}$, Hervé Philippe ${ }^{2,3}$ and Denis Baurain ${ }^{1 *}$ (B)
}

\begin{abstract}
Objectives: Identifying orthology relationships among sequences is essential to understand evolution, diversity of life and ancestry among organisms. To build alignments of orthologous sequences, phylogenomic pipelines often start with all-vs-all similarity searches, followed by a clustering step. For the protein clusters (orthogroups) to be as accurate as possible, proteomes of good quality are needed. Here, our objective is to assemble a data set especially suited for the phylogenomic study of algae and formerly photosynthetic eukaryotes, which implies the proper integration of organellar data, to enable distinguishing between several copies of one gene (paralogs), taking into account their cellular compartment, if necessary.

Data description: We submitted 73 top-quality and taxonomically diverse proteomes to OrthoFinder. We obtained 47,266 orthogroups and identified 11,775 orthogroups with at least two algae. Whenever possible, sequences were functionally annotated with eggNOG and tagged after their genomic and target compartment(s). Then we aligned and computed phylogenetic trees for the orthogroups with IQ-TREE. Finally, these trees were further processed by identifying and pruning the subtrees exclusively composed of plastid-bearing organisms to yield a set of 31,784 clans suitable for studying photosynthetic organism genome evolution.
\end{abstract}

Keywords: Orthology, Phylogenomics, Algae, CASH, Proteomes, Eukaryotic evolution, Contamination, Organelles, Endosymbiotic gene transfer (EGT), Horizontal or lateral gene transfer (HGT/LGT), Kleptoplasty

\section{Objective}

Our main objective is to analyse the phylogenetic origin of plastid-targeted genes in complex algae [1-3] in a fully automated fashion. To do so, we designed and developed a series of strategies and tools around a large-scale single-gene tree analysis pipeline. The first step was to build alignments of orthologous sequences with OrthoFinder, a high accuracy orthogroup inference algorithm [4]. We focused on top-quality proteomes, especially with high completeness, which is essential to obtain the most complete and balanced OGs possible $[5,6]$. In order to maximize completeness and to facilitate the phylogenetic analysis, we complemented beforehand the proteomes having no or only incomplete plastid and/or nucleomorph sequences. Then we processed the resulting OGs, first by isolating the OGs containing photosynthetic organisms, and second by sorting out gene copies shared by plastid-bearing algae from their paralogs. To this end, we built trees using IQ-TREE [7] and used our own tool (tree-clan-splitter.pl) to detect and prune the subtree(s) of interest.

\footnotetext{
*Correspondence: denis.baurain@uliege.be

1 InBioS - PhytoSYSTEMS, Eukaryotic Phylogenomics, University of Liège,

Liège, Belgium

Full list of author information is available at the end of the article
}

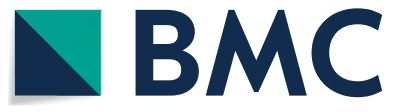

C The Author(s) 2021. This article is licensed under a Creative Commons Attribution 4.0 International License, which permits use, sharing, adaptation, distribution and reproduction in any medium or format, as long as you give appropriate credit to the original author(s) and the source, provide a link to the Creative Commons licence, and indicate if changes were made. The images or other third party material in this article are included in the article's Creative Commons licence, unless indicated otherwise in a credit line to the material. If material is not included in the article's Creative Commons licence and your intended use is not permitted by statutory regulation or exceeds the permitted use, you will need to obtain permission directly from the copyright holder. To view a copy of this licence, visit http://creativeco mmons.org/licenses/by/4.0/. The Creative Commons Public Domain Dedication waiver (http://creativecommons.org/publicdomain/ zero/1.0/) applies to the data made available in this article, unless otherwise stated in a credit line to the data. 


\section{Data description}

We collected 73 top-quality eukaryotic proteomes (i.e., conceptually translated genomes; Data file 1, Data set 1 , Data set 2) with high completeness (Data file 2) $[5,6]$ and low contamination levels (Data set 3 ) $[8,9]$ (Table 1 ). Those were selected to be taxonomically diverse, covering all photosynthetic phyla $[10,11]$, along with some non-photosynthetic organisms to be used as beacons by our clan-identifying algorithm. Those proteomes were complemented with organellar (i.e., plastid and nucleomorph) proteins if they were partly or fully missing in the original source. Hence, 16 were complemented with plastid proteomes whereas two were complemented with nucleomorph proteomes. All proteomes (complemented or not) were dereplicated with CD-HIT [12]. In addition, we used tag-loc-ids.pl, a custom tool designed to tag sequence identifiers according to their encoding genome and cellular localization, such as nuclear-encoded-and-plastid-targeted (nucpt\#), nuclearencoded-periplastid-compartment-targeted (nuppct\#), plastid-encoded-plastid-targeted (cpcpt\#), nucleomorphencoded ( $\mathrm{nm} \#)$, and mitochondrion-encoded ( $\mathrm{mt} \#)$, to facilitate subsequent phylogenetic analyses. Then, we used OrthoFinder [4] for orthology inference, which resulted in 47,266 OGs (Data file 3, Data set 4), composed of two or more sequences belonging to eleven main taxonomic groups (according to NCBI Taxonomy [13]), either classified as "primary algae" (Glaucocystophyceae, Rhodophyta, Viridiplantae) or "complex algae" (Apicomplexa, Colpodellida, Dinophyceae, Cryptophyceae, Euglenozoa, Ochrophyta (including Pelagophyceae), Haptophyta, and Chlorarachniophyceae). Hence, OGs were tabulated into three different categories: "two-algae" (at least one complex alga from two different groups or at least one complex alga and one primary alga, $\mathrm{n}=11,775)$, "onealga" (at least one alga, $\mathrm{n}=18,844$ ) and "zero-algae" (no algae, $n=16,647)$ using the script classify-mcl-out.pl. In order to address the issue of multiple-copy genes (paralogs), we developed a strategy to isolate subtrees ("clans") of interest, i.e., including only plastid-bearing organisms. Briefly, we computed trees for the 11,775 "two-algae" OGs when possible (i.e., $\geq 3$ sequences, $n=11,499$ ) with IQ-TREE [7] and developed a tool for identifying and pruning subtrees fulfilling user-specified taxonomic filters (tree-clan-splitter.pl). This way, we obtained 31,784 "photosynthetic" clans (Data set 5) only composed of plastid-bearing organisms (including species with a nonphotosynthetic plastid, such as Plasmodium falciparum). Additionally, we provide detailed annotation reports obtained with eggNOG [14].

\section{Limitations}

- Occasionally, organellar genome sequences are from a different strain than the nucleus data; it could be an issue if we were trying to resolve relationships between close relatives of the same lineage. Nonetheless, it is not the case here, since the major endosymbiotic-like events we are tracking occurred most certainly between distinct lineages.

- The way we handle the tagging overwrites the information about potential NUMTs, NUNMs and NUPTs; this means that if a gene existed in both genomic compartments (nucleus and organelle) we always retained the organellar counterpart.

Table 1 Overview of data files/data sets

\begin{tabular}{llll}
\hline Label & Name of data file/data set & File types (file extension) & Data repository and identifier (DOl or accession number) \\
\hline Additional file 1 & Methods & PDF file (.pdf) & Figshare https://doi.org/10.6084/m9.figshare.13604102.v3 [18] \\
Data file 1 & Taxonomic sampling & Image file (.png) & Figshare https://doi.org/10.6084/m9.figshare.13603511.v1 [19] \\
Data set 1 & Proteome set description & Text files (.csv,.html) & Figshare https://doi.org/10.6084/m9.figshare.13113893.v1 [20] \\
Data set 2 & Proteome files & FASTA files (.tar.gz) & Figshare https://doi.org/10.6084/m9.figshare.13573424.v2 [21] \\
Data file 2 & BUSCO report & Text file (.csv) & Figshare https://doi.org/10.6084/m9.figshare.13235045.v1 [22] \\
Data set 3 & Forty-Two reports and configu- & Text files (.tsv,.Csv.yaml) & Figshare https://doi.org/10.6084/m9.figshare.13235063.v3 [23] \\
& ration files & & \\
Data file 3 & Orthogroup properties & Image file (.pdf) & Figshare https://doi.org/10.6084/m9.figshare.13312622.v1 [24] \\
Data set 4 & Orthogroups & FASTA files, YAML configura- & Figshare https://doi.org/10.6084/m9.figshare.13573658.v3 [25] \\
& tion file (.tar.gz) & \\
Data set 5 & Clans & FASTA files (.tar.gz) & Figshare https://doi.org/10.6084/m9.figshare.13573415.v1 [26] \\
Data file 4 & Organelle database & Text file (.tsv) & Figshare https://doi.org/10.6084/m9.figshare.13246841.v1 [27] \\
Data file 5 & Plastid-targeted proteins & Spreadsheet (.xlsx) & Figshare https://doi.org/10.6084/m9.figshare.13246784.v1 [28] \\
Data file 6 & eggNOG OG annotations & Text file (.tsv) & Figshare https://doi.org/10.6084/m9.figshare.13415048.v1 [29] \\
Data file 7 & eggNOG clan annotations & Text file (.tsv) & Figshare https://doi.org/10.6084/m9.figshare.13415060.v1 [30] \\
\hline
\end{tabular}


- Only a few of the nucleus-encoded-and-plastidtargeted proteins (nucpt\#) were identified by proteomics (e.g., in P. falciparum) [17]; the remaining are the results of in silico predictions $[15,16]$, which are less reliable than proteomic experiments.

\begin{abstract}
Abbreviations
OGs: Orthologous groups or orthogroups; NUMTs: Nuclear mitochondrial DNAs; NUNMs: Nucleomorph-derived DNAs; NUPTs: Nuclear plastid DNAs.
\end{abstract}

\section{Acknowledgements}

We are grateful to Ugo Cenci for sharing the protein sequences of Goniomonas avonlea (Cenci U, Sibbald SJ, Curtis BA, Kamikawa R, Eme L, Moog D, et al. Nuclear genome sequence of the plastid-lacking cryptomonad Goniomonas avonlea provides insights into the evolution of secondary plastids. BMC Biology (2018) 16:137. https://doi.org/10.1186/s12915-018-0593-5).

\section{Authors' contributions}

MVV and DB designed the experiments, MVV performed all the computational analyses and drew the figures, MVV and DB wrote the manuscript. HP substantively revised the work. All authors read and approved the final manuscript.

\section{Funding}

Mick Van Vlierberghe was a FRIA fellow of the FRS-FNRS (National Fund for Scientific Research of Belgium). Computational resources were provided through two grants to Denis Baurain (University of Liège "Crédit de démarrage 2012" SFRD-12/04; FRS-FNRS "Crédit de recherche 2014"CDR J.0080.15).

\section{Availability of data and materials}

All data generated or analysed during this study are publicly available in the figshare repository (https://doi.org/10.6084/m9.figshare.13604102.v3, https:// doi.org/10.6084/m9.figshare.13603511.v1, https://doi.org/10.6084/m9.figsh are.13113893.v1, https://doi.org/10.6084/m9.figshare.13573424.v2, https:// doi.org/10.6084/m9.figshare.13235045.v1, https://doi.org/10.6084/m9.figsh are.13235063.v3, https://doi.org/10.6084/m9.figshare.13312622.v1, https:// doi.org/10.6084/m9.figshare.13573658.v3, https://doi.org/10.6084/m9.figsh are.13573415.v1, https://doi.org/10.6084/m9.figshare.13246841.v1, https:// doi.org/10.6084/m9.figshare.13246784.v1, https://doi.org/10.6084/m9.figsh are.13415048.v1, https://doi.org/10.6084/m9.figshare.13415060.v1). Please see Table 1 and reference list [18-30] for details and links to the data.

\section{Declarations}

\section{Ethics approval and consent to participate}

Not applicable.

\section{Consent for publication}

Not applicable.

\section{Competing interests}

The authors declare that they have no competing interests.

\section{Author details}

${ }^{1}$ InBioS - PhytoSYSTEMS, Eukaryotic Phylogenomics, University of Liège, Liège, Belgium. ${ }^{2}$ Station D’Ecologie Théorique Et Expérimentale de Moulis, UMR CNRS 5321, Moulis, France. ${ }^{3}$ Département de Biochimie, Centre RobertCedergren, Université de Montréal, Montréal, Québec, Canada.

Received: 18 January 2021 Accepted: 2 April 2021

Published online: 17 April 2021

\section{References}

1. Petersen J, Teich R, Brinkmann H, Cerff R. A "green" phosphoribulokinase in complex algae with red plastids: evidence for a single secondary endosymbiosis leading to haptophytes, cryptophytes, heterokonts, and dinoflagellates. J Mol Evol. 2006;62:143-57.

2. Teich R, Zauner S, Baurain D, Brinkmann H, Petersen J. Origin and distribution of Calvin cycle fructose and sedoheptulose bisphosphatases in plantae and complex algae: a single secondary origin of complex red plastids and subsequent propagation via tertiary endosymbioses. Protist. 2007;158:263-76. https://doi.org/10.1016/j.protis.2006.12.004.

3. Sibbald SJ, Archibald JM. Genomic Insights into Plastid Evolution. Genome Biol Evol. 2020;12:978-90.

4. Emms DM, Kelly S. OrthoFinder: solving fundamental biases in whole genome comparisons dramatically improves orthogroup inference accuracy. Genome Biol. 2015;16:157. https://doi.org/10.1186/ s13059-015-0721-2.

5. Simão FA, Waterhouse RM, loannidis P, Kriventseva EV, Zdobnov EM. BUSCO: Assessing genome assembly and annotation completeness with single-copy orthologs. Bioinformatics. 2015;31:3210-2.

6. Waterhouse RM, Seppey M, Simao FA, Manni M, loannidis P, Klioutchnikov $G$, et al. BUSCO applications from quality assessments to gene prediction and phylogenomics. Mol Biol Evol. 2018:35:543-8.

7. Nguyen LT, Schmidt HA, Von Haeseler A, Minh BQ. IQ-TREE: A fast and effective stochastic algorithm for estimating maximum-likelihood phylogenies. Mol Biol Evol. 2015;32:268-74.

8. Simion P, Philippe H, Baurain D, Jager M, Richter DJ, Di Franco A, et al. A large and consistent phylogenomic dataset supports sponges as the sister group to all other animals. Curr Biol. 2017;27:958-67. https://doi. org/10.1016/J.CUB.2017.02.031

9. Irisarri I, Baurain D, Brinkmann H, Delsuc F, Sire JY, Kupfer A, et al. Phylotranscriptomic consolidation of the jawed vertebrate timetree. Nat Ecol Evol. 2017;1:1370-8.

10. Blaby-Haas CE, Merchant SS. Comparative and functional algal genomics. Annu Rev Plant Biol. 2019;70:605-38. https://doi.org/10.1146/annurevarplant-050718-095841.

11. Hanschen ER, Starkenburg SR. The state of algal genome quality and diversity. Algal Res. 2020;50:101968. https://doi.org/10.1016/j.algal.2020. 101968.

12. Li W, Godzik A. Cd-hit: a fast program for clustering and comparing large sets of protein or nucleotide sequences. Bioinformatics. 2006;22(13):1658-9.

13. Schoch CL, Ciufo S, Domrachev M, Hotton CL, Kannan S, Khovanskaya R, et al. NCBI Taxonomy: a comprehensive update on curation, resources and tools. Database (Oxford). 2020;2020:1-21.

14. Huerta-Cepas J, Forslund K, Coelho LP, Szklarczyk D, Jensen LJ, Von Mering $C$, et al. Fast genome-wide functional annotation through orthology assignment by eggNOG-mapper. Mol Biol Evol. 2017;34:2115-22.

15. Dorrell RG, Gile G, McCallum G, Méheust R, Bapteste EP, Klinger CM, et al. Chimeric origins of ochrophytes and haptophytes revealed through an ancient plastid proteome. Elife. 2017;6:1-45.

16. Novák AMG, Orcid V, Füssy Z, Ebenezer TE, Dobáková EL, Eliáš M. Metabolic quirks and the colourful history of the Euglena gracilis secondary plastid. 2019:44:0-2.

17. Boucher MJ, Ghosh S, Zhang L, Lal A, Jang SW, Ju A, et al. Integrative proteomics and bioinformatic prediction enable a high-confidence apicoplast proteome in malaria parasites. PLOS Biol. 2018;16:e2005895. https://doi.org/10.1371/journal.pbio.2005895.

18. Van Vlierberghe M, Philippe H, Baurain D. Supplementary file 1-Methods. 2021. Figshare. https://doi.org/10.6084/m9.figshare.13604102.v3.

19. Van Vlierberghe $M$, Philippe $H$, Baurain D. Data file 1-Taxonomic sampling. 2021. Figshare. https://doi.org/10.6084/m9.figshare.13603511.v1.

20. Van Vlierberghe M, Philippe H, Baurain D. Data set 1-Proteome set description. 2021. Figshare. https://doi.org/10.6084/m9.figshare.13113 893.v1.

21. Van Vlierberghe M, Philippe H, Baurain D. Data set 2-Proteome set. 2021. Figshare. https://doi.org/10.6084/m9.figshare.13573424.v2.

22. Van Vlierberghe M, Philippe H, Baurain D. Data file 2-BUSCO report. 2021. Figshare. https://doi.org/10.6084/m9.figshare.13235045.v1.

23. Van Vlierberghe $M$, Philippe $H$, Baurain D. Data set 3-Forty-two reports and configuration files. 2021. Figshare. https://doi.org/10.6084/m9.figsh are.13235063.v3. 
24. Van Vlierberghe $M$, Philippe $H$, Baurain D. Data file 3-Orthogroup properties. 2021. Figshare. https://doi.org/10.6084/m9.figshare.13312622.v1.

25. Van Vlierberghe M, Philippe H, Baurain D. Data set 4-Orthogroups. 2021. Figshare. https://doi.org/10.6084/m9.figshare.13573658.v3.

26. Van Vlierberghe M, Philippe H, Baurain D. Data set 5-Clans. 2021. Figshare. https://doi.org/10.6084/m9.figshare.13573415.v1.

27. Van Vlierberghe M, Philippe H, Baurain D. Data file 4-Organelle database. 2021. Figshare. https://doi.org/10.6084/m9.figshare.13246841.v1.

28. Van Vlierberghe M, Philippe H, Baurain D. Data file 5-Plastid-targeted proteins. 2021. Figshare. https://doi.org/10.6084/m9.figshare.13246784. v1.
29. Van Vlierberghe $M$, Philippe $H$, Baurain D. Data file 6-eggNOG OG annotations. 2021. Figshare. https://doi.org/10.6084/m9.figshare.13415048.v1.

30. Van Vlierberghe M, Philippe $H$, Baurain D. Data file 7-eggNOG clan annotations. 2021. Figshare. https://doi.org/10.6084/m9.figshare.13415060.v1.

\section{Publisher's Note}

Springer Nature remains neutral with regard to jurisdictional claims in published maps and institutional affiliations.
Ready to submit your research? Choose BMC and benefit from:

- fast, convenient online submission

- thorough peer review by experienced researchers in your field

- rapid publication on acceptance

- support for research data, including large and complex data types

- gold Open Access which fosters wider collaboration and increased citations

- maximum visibility for your research: over $100 \mathrm{M}$ website views per year

At BMC, research is always in progress.

Learn more biomedcentral.com/submissions 